\title{
THE RADIATION ENVIRONMENT IN AND NEAR HIGH GRADIENT RF CAVITIES
}

\author{
J. Norem, ANL, Argonne, IL, 60439 \\ S. Geer, A. Moretti, M Popovic, Fermilab, Batavia, IL, 60510 \\ N. Solomey, IIT, Chicago, IL, 60616 \\ L. Ducas, University of Illinois, Urbana-Champaign, IL,61801
}

\begin{abstract}
The radiation environment in and near high gradient $\mathrm{rf}$ cavities is very important for the instrumentation of the MUCOOL experiment, since large fluxes of $x$ rays and dark current electrons can interfere with the operation of the muon detectors. We have measured the $\mathrm{x}$ ray and dark current spectra from a single cell, $1.3 \mathrm{GHz}$, and are beginning to make more extensive measurements of a multicelled $805 \mathrm{MHz}$ cavity. The results are consistent with electron field emission, bremsstrahlung and photon absorption/scattering. We discuss ways of minimizing this background and the scaling of these results to other cavities.
\end{abstract}

\section{INTRODUCTION}

Ionization cooling of muons requires repeated $\mathrm{rf}$ acceleration sections interspersed with liquid hydrogen absorbers to remove the transverse momentum from the beam. We have completed measurements at a $1.3 \mathrm{GHz}$ cavity at Argonne, and are taking data in an $805 \mathrm{MHz}$ cavity in Lab G at Fermilab and the ATF at Brookhaven. In addition we have looked at data from LEP cavities and the Fermilab 200 / 800 MHz linac system.

\section{X RAY PRODUCTION}

Dark current electrons are produced in rf cavities by field emission, as described by Fowler and Nordheim[1] in 1928 and experimentally checked in 1953[2]. The electrons produced by this mechanism can be further amplified by multipactoring. These mechanisms are well described in Padamsee, Knobloch and Hays[3].

The electrons produced by this mechanism will collide with the walls of the cavity, vacuum chamber and external environment, producing bremsstrahlung radiation. Depending on the energy of the electrons, this bremsstrahlung may be of sufficient energy to cause further showering and photon production. At low energy, the probability for photon production is proportional to the range of the electron divided by the radiation length of the material, so very low energy electrons do not produce many photons.

The subsequent fate of the photons depends on their energy. Low energy photons tend to be absorbed with a very large cross section into atoms, ionizing the atoms. At higher energies, this photoabsorption cross section becomes smaller and photons can only undergo Compton scattering. One should remember that while the photons are degraded in energy, perhaps enough to be absorbed by the photoabsorption, Compton scattering diffuses photons rather than absorbs them and there is no attenuation. At high energies, the photons can produce pairs, which are another source of bremsstrahlung photons and potential showers. These processes have been modeled using EGS4[4].

\section{1.3 GHZ RESULTS}

At Argonne, a range/absorber array was used to look at the radiation spectrum from a single cell cavity. The method used is shown in Figure 1. The method works as an absorber array for photons, and simultaneously, a range telescope for electrons, unfortunately causing some confusion. The method is described in Figure 1. The detectors were TLDs, which have a linear response over the expected energy range[5].

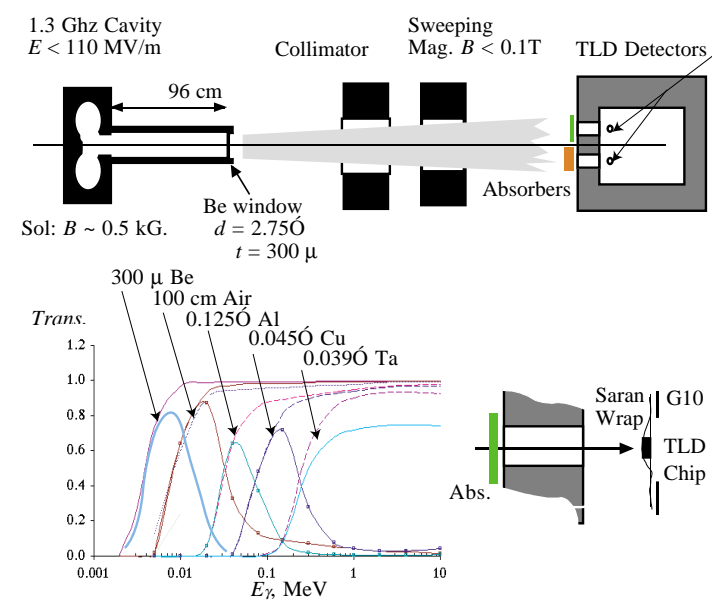

Figure 1, The apparatus used with the $1.3 \mathrm{GHz}$ cavity.

Data were taken at a distance of $12 \mathrm{~m}$ from the cavity at both zero degrees and 90 degrees; the zero degree line had a sweeping magnet in it to help determine the composition of the beam. Figures 2 and 3 show the data assuming all the radiation consisted of photons.

The EGS Monte Carlo was used predict the spectrum of photons which might be expected to be produced by the 1 $2 \mathrm{MeV}$ dark current electrons, and this curve was plotted on the graph. Because the photon energy distribution is dominated by the maximum energy of electrons at the top, the photoabsorbtion at the bottom, and a large and somewhat isotropic Compton photon flux in the range of 
$1 \mathrm{MeV}$, the gross features of the photon spectrum are fairly simple.

Assuming that the right most part of the experimental data is from $x$ rays (which have a fairly flat spectrum), and the left most points are due to electrons, one can derive the spectrum of electrons that would cause the low energy peak in the zero degree data. This spectrum is shown in Figure 4.

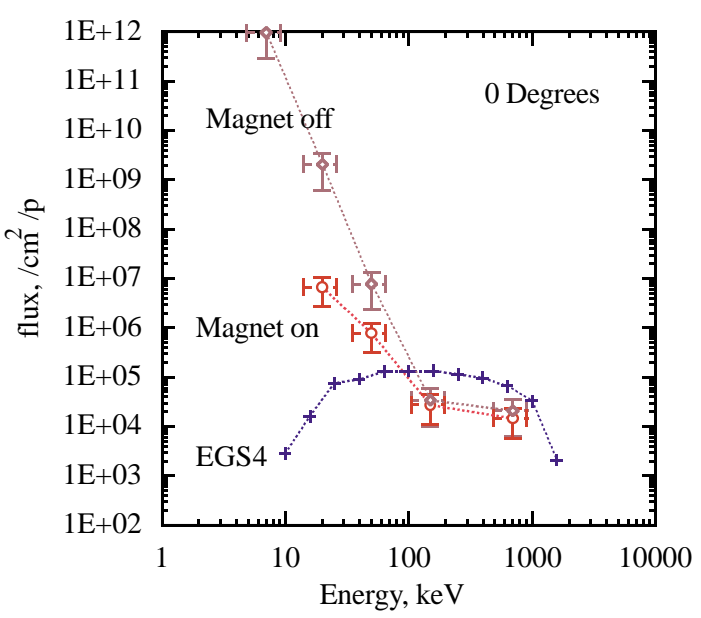

Figure 2, The results at zero degrees.

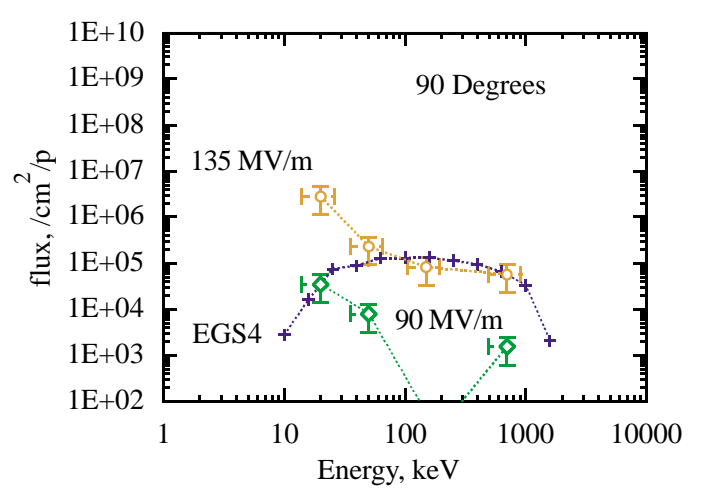

Figure 3, The results at 90 degrees.

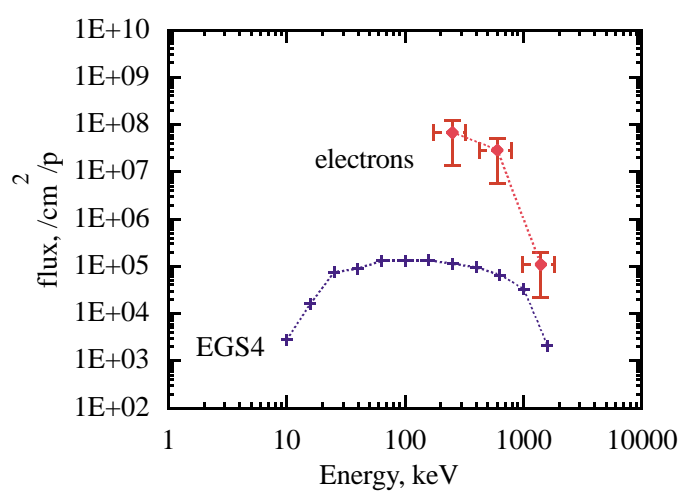

Figure 4, The possible spectrum of electrons and photons produced at zero degrees.

The use of the seeping magnet provides additional identification of the electron signal, although the low energy electrons would be expected to constitute a fairly diffuse beam. The electron flux calculated in this way is grossly compatible with measurements of dark current made with electron probes.[6]

Data from this cavity and others show a strong dependence of the radiation produced on the electric field. Fitted results show that the radiation dose measured at 90 degrees inside the shielding show that radiation dose is proportional to $E^{9.6}$, where $E$ is the electric field. This behavior is consistent with Fowler-Nordheim field emission. The complete results of this work have been submitted to NIM[7].

\section{MHZ RESULTS}

As part of the MUCOOL experiment, a six cell standing wave cavity was constructed, and made compatible with pair of superconducting coils in a Helmholtz geometry. These coils can be energized either in a parallel or bucking geometry, and produce a field in the range of $5 \mathrm{~T}$. This facility has been installed in Lab G and should reach full field during this conference.

\section{OTHER MEASUREMENTS}

The mechanisms discussed here would argue that all measurements of dark currents and $\mathrm{x}$ rays should be, to some extent, similar. The most complete studies of dark current production have been made as part of the superconducting rf program, and these have been collected in reference [3]. These cavities have very carefully prepared surfaces and many parameters may be different.

Other systematic measurements have been made as part of the LEP superconducting rf development program[4]. These data, for $\mathrm{cw}$ superconducting cavities running at an accelerating field of about $6 \mathrm{MV} / \mathrm{m}$, also find that radiation dose is proportional to $E^{9.6}[8]$.

Recently, silicon fiber detectors have been used at the BNL ATF to measure the radiation flux from the rf photocavity. Single fibers were wrapped around the beam pipe, roughly a meter downstream, and the output signal was recorded. The data showed the same strong dependence on the electric field as was seen in other measurements, in this case $E^{10.6}$.

\section{COMMENTS}

Since the dark currents are a by product of the high electric field gradient produced in cavities run near their maximum field gradient, they are difficult to eliminate. Three methods should be considered: 1) reducing the electric field gradient, 2) coating the cavities with some material with a low secondary electron yield, and 3) using a geometry where the dark current electrons tend to miss the detectors or produce bremsstrahlung in a well shielded place.

Since the cavities and power supplies are very expensive, it is usually desirable to operate the system in a mode where it produces the maximum field gradient 
possible. Thus, reducing the field gradient is not usually an economic solution.

A number of materials have been considered for coatings of rf cavities. These materials have been shown to reduce dark currents by many orders of magnitude. There is not much experience with them in a working accelerator environment however, and perhaps they could be more carefully evaluated[10].

The geometry of the cavity / absorber / detector to some extent should determine the radiation environment. It seems likely that the SFOFO geometry used in current muon cooling simulations may exacerbate the problem since the magnetic field lines would guide dark current electrons toward the detectors. On the other hand, the double flip geometry, which is essentially a long solenoid, would prevent dark current electrons from reaching the detectors, perhaps resulting in lower radiation flux. Dark current electrons tend to be very low energy and can be ranged out in very thin absorbers. It has been proposed that the LH2 absorbers will filter these to a significant extent, since the $\mathrm{dE} / \mathrm{dx}$ losses of these absorbers will be equal to the energy gain of the muons, and thus, roughly equal to the energy of the dark current electrons[11]. Hydrogen has a very long radiation length so $\mathrm{x}$ ray production will be minimal.

\section{CONCLUSIONS}

Large fluxes of $\mathrm{x}$ rays and dark currents are produced in rf cavities and these will complicate measurements of single particle detectors nearby. Preliminary data are consistent with the primary mechanisms being field emission, bremsstrahlung and photon absorption. Additional work is necessary to develop beam diagnostics that will work in this environment.

\section{ACKNOWLEDGEMENTS}

This work was supported in part by the U.S. DOE, Office of High Energy Physics, and by the Illinois Board of Higher Education. W. Gai and M. Conde of ANL/HEP, T. Leveling of FNAL/ Radiation Safety, G. Geschonke and E. Keil of CERN, S. Henderson of Cornell, and Elwin Dolochek of ANL Health Physics have been very helpful.

\section{REFERENCES}

[1] R. H. Fowler, and L. W. Nordheim, Proc. Roy. Soc. (London) A119, 173,(1928)

[2] W. P. Dyke and J. K. Trolan, Phys. Rev. 89 (1953), 799.

[3] H. Padamsee, J. Knobloch, and T. Hays. RF Superconductivity for Accelerators, John Wiley and Sons, Inc, New York (1998).

[4] http;//ehssun.lbl.gov/egs/egs.html.

[5] G. Shani, Radiation Dosimetry Instrumentation and Methods, CRC Press, Boca Raton (1991).

[6] C. H. Ho, et. al., SSRC/ANL High Current L-Band Single Cell Photocathode RF gun, EPAC 98, Institute of Physics Pub., London (1998), 1441.
[7] J. Norem, A. Moretti, M. Popovic, The Radiation Environment in and near High Gradient RF Cavities, to be published in Nuclear Inst. and Methods in Physics Research, A (2001).

[8] M. Silari, S. Agosteo, J. C. Gaborit, L. Ulrici, Nucl Instrum. Methods Phys. Res. A, 432 (1999) 1.

[9] K. T. McDonald, H. G. Kirk, X. J. Wang, X-Ray Rates in Scintillating Fibers Placed Near the BNL ATF RF Gun, Princeton Note, Princeton/mm/01-22, (2001).

[10] R. Silberglitt, FM Technologies, Fairfax VA, Private Communication

[11] H. Haseroth, CERN, Private Communication, (2001) 